\title{
Posttranslational modification and beyond: interplay between histone deacetylase 6 and heat-shock protein 90
}

\author{
Ping Liư ${ }^{1 \dagger}$, Ji Xiao ${ }^{1 \dagger}$, Yiliang Wang ${ }^{1}$, Xiaowei Song ${ }^{1}$, Lianzhou Huang ${ }^{1,2}$, Zhe Ren ${ }^{1}$, Kaio Kitazato ${ }^{3 *}$ and \\ Yifei Wang ${ }^{1 *}$ (D)
}

\begin{abstract}
Posttranslational modification (PTM) and regulation of protein stability are crucial to various biological processes. Histone deacetylase 6 (HDAC6), a unique histone deacetylase with two functional catalytic domains (DD1 and DD2) and a ZnF-UBP domain (ubiquitin binding domain, BUZ), regulates a number of biological processes, including gene expression, cell motility, immune response, and the degradation of misfolded proteins. In addition to the deacetylation of histones, other nonhistone proteins have been identified as substrates for HDAC6. Hsp90, a molecular chaperone that is a critical modulator of cell signaling, is one of the lysine deacetylase substrates of HDAC6. Intriguingly, as one of the best-characterized regulators of Hsp90 acetylation, HDAC6 is the client protein of Hsp90. In addition to regulating $\mathrm{Hsp} 90$ at the post-translational modification level, $\mathrm{HDAC} 6$ also regulates $\mathrm{Hsp} 90$ at the gene transcription level. HDAC6 mainly regulates the Hsp90-HSF1 complex through the ZnF-UBP domain, thereby promoting the HSF1 entry into the nucleus and activating gene transcription. The mutual interaction between HDAC6 and Hsp90 plays an important role in the regulation of protein stability, cell migration, apoptosis and other functions. Plenty of of studies have indicated that blocking HDAC6/Hsp90 has a vital regulatory role in multifarious diseases, mainly in cancers. Therefore, developing inhibitors or drugs against HDAC6/Hsp90 becomes a promising development direction. Herein, we review the current knowledge on molecular regulatory mechanisms based on the interaction of HDAC6 and Hsp90 and inhibition of HDAC6 and/or Hsp90 in oncogenesis and progression, antiviral and immune-related diseases and other vital biological processes.
\end{abstract}

Keywords: HDAC6, Hsp90, Client proteins, Acetylation, Drug development

\section{Background}

Post-translational modification (PTM) of proteins is a common form of epigenetic regulation. Acetylation, one of the posttranslational modifications of proteins, plays a powerful role in regulating gene expression, protein

\footnotetext{
*Correspondence: kitazato8@gmail.com; twang-yf@163.com

${ }^{\dagger}$ Ping Liu and Ji Xiao contributed equally to this work

${ }^{1}$ College of Life Science and Technology, Guangzhou Jinan Biomedicine Research and Development Center, Jinan University, Guangzhou, China

${ }^{3}$ Department of Clinical Research Pharmacy, Graduate School of Biomedical Sciences, Nagasaki University, Nagasaki, Japan

Full list of author information is available at the end of the article
}

stability, and enzyme activity (Morgan and Shilatifard 2020). Typically, acetylation consists of histone and nonhistone acetylation modifications. Acetylation modifications of histone proteins regulate chromatin compaction and mediate the epigenetic regulation of gene transcription (Glozak et al. 2005). Nonhistone acetylation modifications are mainly involved in protein degradation and enzyme activity regulation (Narita et al. 2019). The histone deacetylase (HDAC) family is a large family of primary regulators that participate in histone acetylation. In addition, several HDAC members, especially the cytoplasmic protein HDAC6, play a significant role original author(s) and the source, provide a link to the Creative Commons licence, and indicate if changes were made. The images or other third party material in this article are included in the article's Creative Commons licence, unless indicated otherwise in a credit line to the material. If material is not included in the article's Creative Commons licence and your intended use is not permitted by statutory regulation or exceeds the permitted use, you will need to obtain permission directly from the copyright holder. To view a copy of this licence, visit http://creativecommons.org/licenses/by/4.0/. 
(See figure on next page.)

Fig. 1 The protein structure diagram of HDAC6 and mRNA expression in different organs of Homo sapiens. A HDAC6 consists of two functional catalytic domains (DD1 and DD2) and a ZnF-UBP domain (ubiquitin binding domain, BUZ). B HDAC6 mRNA is widely expressed in various organs of Homo sapiens. C Domain architecture and alternative acetylation site of Hsp90 ( $N$, N-terminal binding region; C, C-terminal binding region; M, intermediate connection region)

in the regulation of nonhistone acetylation. HDAC6 is closely related to multiple diseases involved in malignant tumors (Li et al. 2018), pathogen infection (Zhang et al. 2016), cardiovascular and metabolic diseases (Bagchi and Weeks 2019), neurodegenerative diseases and pathological autoimmune responses (Simões-Pires et al. 2013) by regulating the acetylation of nonhistone proteins (Seidel et al. 2015). Specifically, nonhistone proteins have been identified as substrates of HDAC6, including Hsp90, $\alpha$-tubulin, cortactin, Ku70, RIG-I and $\beta$-Catenin (Zheng et al. 2017). Hsp90 is a member of the family of heat shock proteins (HSPs), which perform their molecular chaperone functions to regulate the folding and degradation of client proteins and further affect various biological functions. A large number of studies have reported that HDAC6 is able to deacetylate Hsp90. Simultaneously, HDAC6 is also a client protein of Hsp90, and the protein stability of HDAC6 is modulated by Hsp90.

Due to the significant biological functions of HDAC6 and Hsp90, exploring inhibitors for antitumor and antiviral biological effects has become a high-profile direction for new drug development. Therefore, we review the mutual regulatory pathway, molecular mechanisms, biological functions and related diseases between HDAC6 and Hsp90, as well as research exploring efficient and specific inhibitors or combined moderator applications, providing a reference for comprehensive recognition and an in-depth understanding of the regulatory mechanism between HDAC6 and Hsp90 molecules and innovative drug exploitation.

\section{HDAC6 is a specific member of the histone deacetylase family}

In mammals, HDACs have been phylogenetically classified into four classes based on their homology of accessory domains to histone deacetylases. Class I HDACs include HDAC1, HDAC2, HDAC3 and HDAC8. Class II HDACs are HDAC4, HDAC5, HDAC6, HDAC7, HDAC9 and HDAC10, and according to their sequence homology and domain organization, they are further categorized into class IIa (HDAC4, HDAC5, HDAC7 and HDAC9) and class IIb (HDAC6 and HDAC10). Class III HDACs are made up of Sir2-like deacetylase silent information regulators (Sirt1, Sirt2, Sirt3, Sirt4, Sirt5, Sirt6 and Sirt7) (Ruijter et al. 2003). As a distinct member of HDACs in higher eukaryotes, HDAC6 was first discovered in mice
(Verdel and Khochbin 1999). HDAC6 presents different molecular features and functions from other HDAC family members based on the molecular structure characteristics of two functional catalytic domains (DD1 and DD2) and a $\mathrm{ZnF}$ domain (ubiquitin binding domain, BUZ) (Fig. 1A). In contrast to the nuclear location of other HDAC family members, HDAC6 is a unique deacetylase due to its cytoplasmic localization and more powerful ability to deacetylate proteins than histones and is widely expressed in various normal tissues and organs of Homo sapiens (humans) (Fig. 1B) (Data from https://www.ncbi. nlm.nih.gov/gene/10013). HDAC6 contains a noncatalytically active ubiquitin-bound zinc finger structure (called the ZnF-UBP domain) and a dynamin-binding domain at the carboxyl terminus. HDAC6 deacetylates nonhistone substrates, including $\alpha$-tubulin, Hsp90, cortactin, Ku70, and $\beta$-Catenin (Hubbert et al. 2002; Matsuyama et al. 2002; Selenica et al. 2014). HDAC6 was previously shown to be a pivotal element in the misfolded protein accumulation-induced stress response by coordinating the clearance of protein aggregates through aggresome formation and their autophagic degradation (Boyault et al. 2007; Matthias et al. 2008). Together with the motor protein dynein, HDAC6 carries cytotoxic polyubiquitinylated proteins into autophagosomes (Li et al. 2013). Therefore, the two functional domains perform important but different functions.

The ubiquitination domain (ZnF-UBP) of HDAC6 is conducive to the formation of pressure particle SG, which can be prevented by interfering with the arrangement of microtubules or damaging dynamin. As an antiviral immune complex, stress particle SGs play positive roles in the IFN immune response and can be autophagy targeted. HDAC6 can mediate SG degradation after being recognized by P62/SQSTM1 (Zheng et al. 2020). In addition, due to the specific ubiquitin binding domain, HDAC6 plays a significant role at the intersection of two important signaling pathways in cells: the protein lysine acetylation pathway and the ubiquitin pathway (Boyault et al. 2007). Because of this, HDAC6 can coordinate and respond to intracellular events under different stress stimuli, including the endoplasmic reticulum stress response (ERS) and the unfolded protein response (UPR) (Iwata et al. 2005; Rao et al. 2008). In response to endoplasmic reticulum stress and unfolded proteins, HDAC6 plays a vital role as a coordinating factor in eliminating 
A

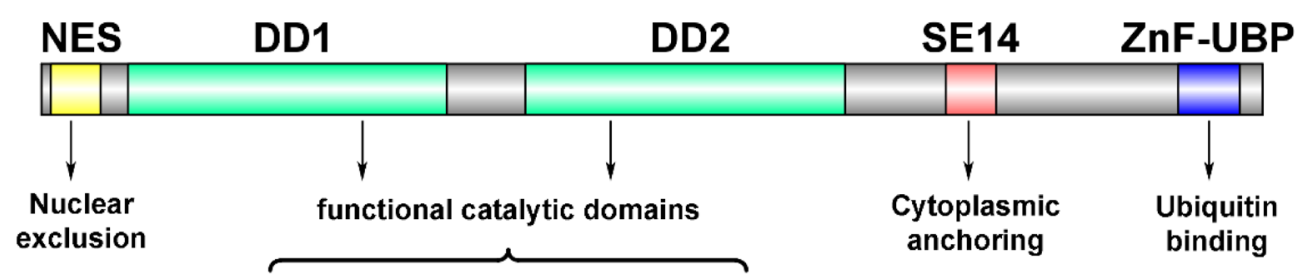

Hsp90, $\alpha$-tubulin, cortactin, Ku70, RIG-I, $\beta$-Catenin

B

HPA RNA-seq normal tissues

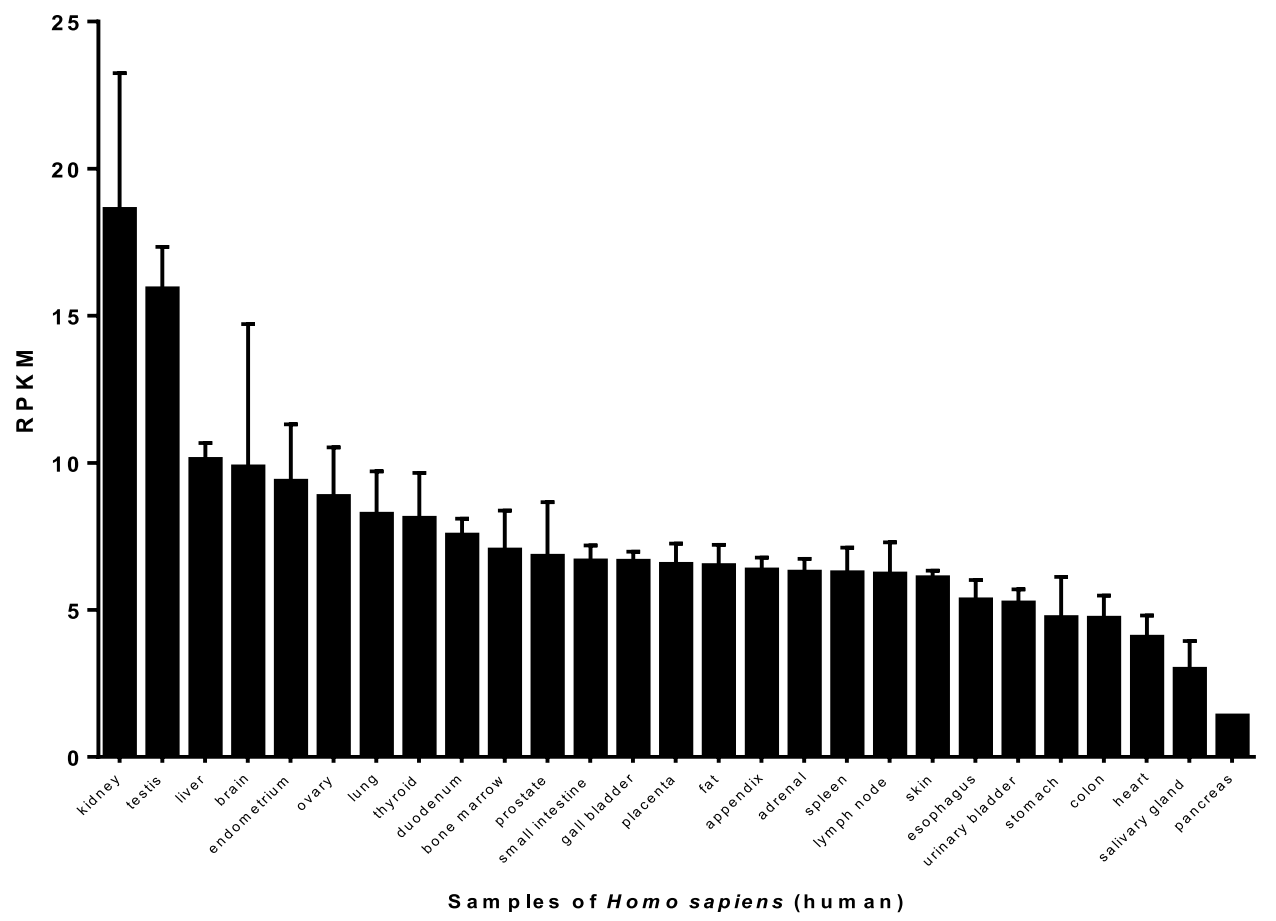

C

\section{client binding domain}

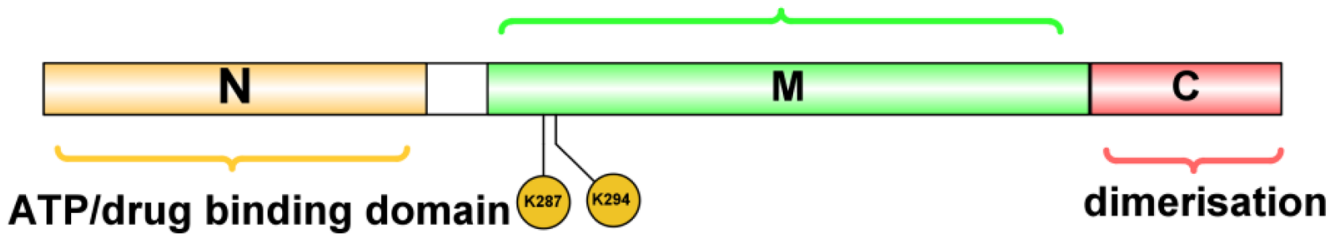


toxicity caused by misfolded or unfolded proteins ( $\mathrm{Li}$ et al. 2017). In addition, HDAC6 plays a key role in the regulation of different cellular stress responses, the results indicate that HDAC6 plays a major regulatory role in different kinds of cellular stress responses. However, the mechanism of HDAC6 in different kinds of cellular stress responses remains unclear (Ryu et al. 2017a).

The ubiquitin-proteasome system (UPS) and autophagy are two important protein degradation pathways in cells. A coordinated and complementary relationship between these two systems is essential for cells to protect themselves from stress. Among them, some important protein regulatory factors, including P62 and HDAC6, play an important role in coordinating with their relationship. The UPS and autophagy are considered key factors in neurodegenerative diseases. Pandy et al. used a Drosophila neurodegenerative disease model to research the relationship between autophagy and HDAC6 and found that HDAC6 autophagy is dependent on the inhibition of the ubiquitin-proteasome pathway, which further reveals the complementary relationship between these two degradation pathways (Zheng and Wang 2010; Pandey et al. 2007). Since the accumulation of protein aggregates is a distinct feature of neurodegenerative diseases, the aggregate-autophagy pathway is expected to be a potential target for the treatment of these diseases (Yan 2014). Notably, the deacetylase activity of HDAC6 is necessary for aggregate formation and can regulate aggregate formation by interacting with other proteins. For example, HDAC6 directly interacts with P62 to regulate aggregate formation, which is localized in ubiquitinated protein aggregates (Yan et al. 2013). However, not all HDAC6 ubiquitin domain-mediated autophagy is associated with its deacetylase activity. HR23B is a biomarker in response to HDAC inhibitors. In the process of the interaction, HR23B expression level determines different cell fates. HDAC inhibitors can lead to apoptosis in cells which expressing high levels of HR23B; In contrast, when HR23B at a low expression level in cells, an HDAC inhibitor promotes autophagy. The specific mechanism of the ZnF-UBP domain of HDAC6 on aggregates and autophagy is as follows: HDAC6 binds to the misfolded proteins of ubiquitination, transports them along the microtubule skeleton to the cell motor protein, transports them to the aggregates, and finally degrades through the lysosomal-autophagy pathway (New et al. 2013; Kalveram et al. 2008). The elucidating of this mechanism will help to enhance the understanding of the molecular mechanism of cancer and provide theoretical basis and unique insights for further exploration of new cancer therapies.

HDAC6 is widely involved in the treatment of various human diseases in the form of inhibitors. HDAC6 inhibitors are gradually being used in clinical drug development and are expected to be a new cancer treatment strategy. For example, Benoy et al. developed three HDAC6 selective inhibitors, ACY-738, ACY-775, and ACY-1215, which have been proven to be effective. These inhibitors have been shown to improve the function of neuromuscular junctions and sensory nerve conduction in gastrocnemius neuromuscle. Notably, ACY-1215 is currently in clinical trials with patients with cancer. This also suggests that inhibition of HDAC6 is a potential strategy for the treatment of CMT2 in axons gastrocnemius (Benoy et al. 2017). In addition, some studies have shown that HDAC6 inhibitors can treat hematological malignancies, which raises the possibility of HDAC inhibitors being administered as potential antitumor drugs. It has been reported that HDAC6 inhibitors have been used in preclinical and clinical studies of lymphoproliferative diseases and have shown certain activity (Cosenza and Pozzi 2018). Currently, more than a dozen HDAC6 inhibitors have been applied in the clinical treatment of cancer, which also proves that HDAC6 is a potential target molecule in clinical drug development.

\section{Characterization of $\mathrm{Hsp} 90$}

Heat shock proteins (HSPs) are highly conserved intracellular proteins in eukaryotic cells and prokaryotic cells. They protect cells mainly by regulating the threedimensional structure of proteins and preventing misfolding and degradation of proteins by the proteasome (Calderwood et al. 2007). HSPs is abundant in cells, accounting for $1 \%-2 \%$ of the total protein, and when subjected to heat, oxidation, chemical stimulation and other stress states, the expression of heat shock proteins will be up-regulated, accounting for about $4 \%-6 \%$ of the total cell proteins (Choudhary et al. 2009). According to the molecular weight, Hsp protein families are mainly divided into five categories, namely small molecule heat shock proteins (12-43KDa), Hsp60, Hsp70 (66-78KDa), Hsp90 (83-94KDa) and Hsp100, among which Hsp60, Hsp70 and Hsp90 are the most widely studied (Lebret et al. 2003). Hsp27 is located in the cytoplasm and its function is mainly to prevent the aggregation of unfolded proteins. Mitochondrial protein Hsp60 is mainly involved in mitochondrial protein folding and assembly. Hsp70 is distributed in mitochondria, nucleus and cytoplasm, and participates in protein transport, anti-apoptosis, and protein degradation (Sreedharan and Why 2016). Hsp90 is mainly composed of four parts: N-terminal binding region, connecting region connecting $\mathrm{N}$-terminal and middle region, middle region and $\mathrm{C}$-terminal binding region (Fig. 1C). In mammalian cells, there are two or more genes encoding cytosolic Hsp90 homologs (Chen et al. 2006). HSP90A is a cytosolic family protein that includes Hsp90 $\alpha$ and Hsp90 $\beta$, which are inducible 
and constitutively expressed forms, respectively (Chen et al. 2005). As an ATP-dependent molecular chaperone, Hsp90 plays a crucial adjuvant role in the process of cellular protein synthesis, correct folding, configuration stability, assembly, transportation and degradation.

According to the role of molecular chaperones in protein folding, there are three main folding pathways: (1) spontaneous chaperone-independent folding; (2) folding assisted by repetitive Hsp70 cycles; and (3) folding by Hsp70-Hsp90 cascades. Among them, helper chaperones such as STI1/Hop play an important role in the Hsp70-Hsp90 chaperone cascade, which promotes the interaction between Hsp70 and Hsp90 by binding their C-termini. Most importantly, Hsp90 has two distinct functions in protein folding: an older, evolutionarily conserved function in protein folding downstream of HSP70 that is independent of the helper chaperone. Second, in the complex and intricate signal network regulation of eukaryotic cytoplasm, it is precisely regulated by many cochaperones (Morán Luengo et al. 2019). Advances in the mechanisms of molecular chaperones and the development of Hsp90 inhibitors may be conducive to stimulate a new generation of therapies that are critical not only for cancer but also for other protein misfolding diseases such as neurodegenerative diseases.

In addition to being molecular chaperones, heat shock proteins also play crucial roles in the activation of the heat shock response. The unfolded and/or misfolded proteins that have accumulated due to cellular stress can be replaced by activation of the heat shock response (HSR). Since Hsp90 is the main regulator of HSR, the regulation of Hsp90 by small molecules represents a therapeutic approach to complications of the peripheral nervous system and central neuropathy (Chaudhury et al. 2021). In addition, Hsp90 plays an important role in a variety of human diseases, such as cancer, inflammation, Alzheimer's disease, Parkinson's disease, and diseases related to protein misfolding. Therefore, Hsp90 is expected to become a new target molecule for drug development, and many of its client proteins are being used as targets for carcinoma therapy in clinical practice (Table 1). However, rather than using the client protein of Hsp90 as a target molecule, it would be better to use Hsp90 as a direct target for tumor therapy, thus achieving targeted therapy. Clinical studies have shown that Hsp90 is significantly positive in patients with early nasopharyngeal carcinoma, and the mechanism may be related to the effect of Hsp90 on the growth and proliferation of human nasopharyngeal carcinoma cells, suggesting that Hsp90 can be used as a marker for early screening of nasopharyngeal carcinoma (Liu et al. 2019). 17-DMAG is a semisynthetic derivative of 17-AAG, and the class I clinical drug Hsp90 has entered clinical trials and can be used alone or in combination with other effective anticancer drugs to optimize the anticancer effect. It is widely used in the treatment of leukemia, melanoma, breast cancer, lymphoma, liver cancer, lung cancer, multiple myeloma and other diseases (Mellatyar et al. 2018). Currently, an increasing number of studies have reported the development of Hsp90-targeted drugs with high specificity, strong potency and similar drug properties, indicating that they are very likely to have important clinical application value (Banerjee et al. 2020).

\section{HDAC6 modulates Hsp90 client proteins by deacetylase activity}

The biological activity of $\mathrm{Hsp} 90$ is regulated by multiple pathways including acetylation. The acetylation sites mapped to K294 (K295 in the mouse ortholog protein) and K287 in Hsp90 $\alpha$ and Hsp90ß, respectively (Scroggins et al. 2007) (Fig. 1C). Acetylation at K69 or K294 reduces ATP binding to Hsp90 and decreases Hsp90 binding to its cochaperones and client proteins. HDAC6 and Hsp90 are present in a cytoplasmic complex, and HDAC6 inhibition leads to Hsp90 acetylation and disruption of its key chaperone functions. HDAC6, which is the deacetylase of Hsp90, thereby leading to hyperacetylation of Hsp90, specifically impairs the chaperone function of Hsp90 by decreasing the affinity of Hsp90 for ATP and client proteins and thus promoting the polyubiquitination and subsequent degradation of Hsp90 client proteins, such as GR, ER $\alpha$, AR, HIF-1 $\alpha$, VEGFRs, AhR, Her2, Racl, and EGFR (Fig. 2). HDAC6 also controls mitochondrial metabolic activity partly through deacetylation of Hsp90 (Kamemura et al. 2012). In addition, some studies have shown that hindering HDAC6 can not only induce the acetylation modification of $\mathrm{Hsp} 90$ but can also directly cause Hsp90 fragmentation and then enhance microglial activation and migration (Tsai et al. 2015; Gao et al. 2007). In view of the large number of client proteins of Hsp90, studies have directly aimed to suppress Hsp90 or affect the function of Hsp90 by regulating HDAC6. In addition to the straightforward regulatory signal pathways and biological function changes of interest, the significant and valuable $\mathrm{Hsp} 90$ regulation process that impacts various biological phenomena also needs to be closely monitored to ensure that the discovery of biological phenomena is one of the main ways of regulating Hsp90.

\section{HDAC6 is regulated by $\mathrm{Hsp} 90$ as a client protein}

As mentioned above, HDAC6 can acetylate Hsp90 and affect the stability of its client proteins. Interestingly, several lines of evidence indicate that HDAC6 is one of the client proteins of Hsp90 (Fig. 2). HDAC6 protein degradation is regulated by the Hsp90 chaperone function in 


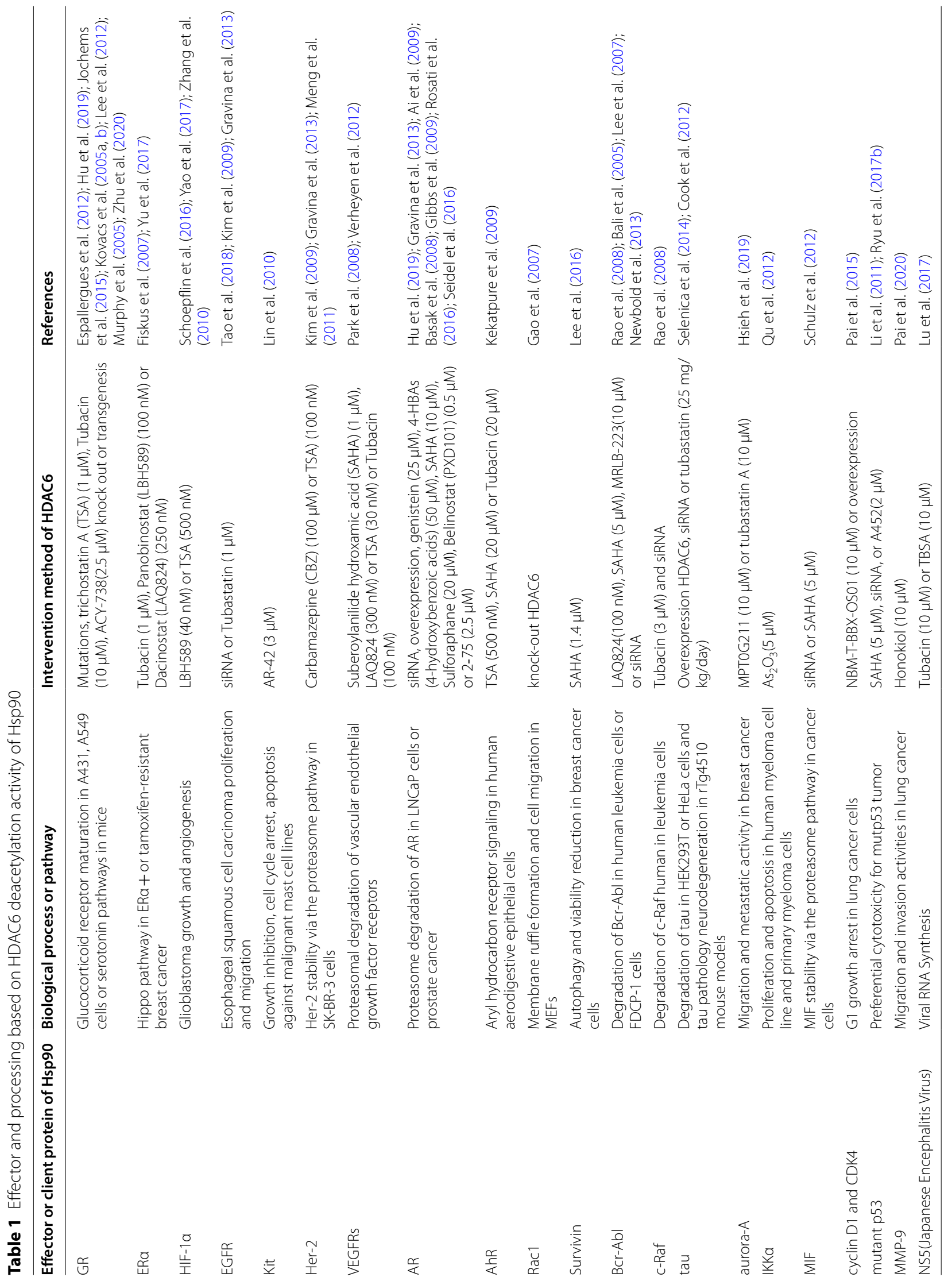




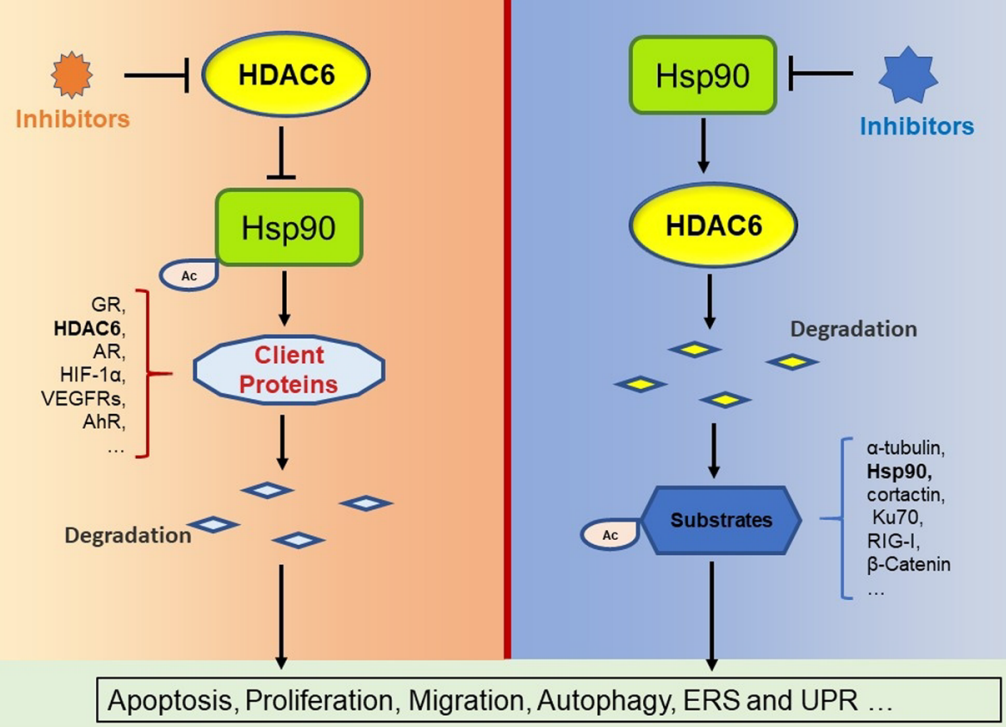

Fig. 2 Interplay between HDAC6 and Hsp90

turn in K562 cells and primary acute myeloid leukemia (AML) (Rao et al. 2008). AT13387, a ground-breaking Hsp90 inhibitor, can restrain Hsp90 molecular chaperone function, downregulate HDAC6 and then increase the acetylation and stabilization of $\alpha$-tubulin, ultimately suppressing nasopharyngeal carcinoma (NPC) oncogenesis and progression (Chan et al. 2013). In conclusion, there is a mutual regulatory relationship between HDAC6 and HSP90, and a thorough understanding of their regulatory mechanism will be helpful for the further development of new clinical drug therapy.

\section{HDAC6 regulates Hsp90 by its ZnF-UBP}

The ZnF-UBP domain of HDAC6 interacts with a variety of proteins, enzymes and molecular chaperones and plays a key regulatory role in a variety of human diseases. The E3 ubiquitin ligase TRIM50 promotes the formation and degradation of ubiquitinated proteins through its interaction with HDAC6 (Fusco et al. 2012), and the Cullin3 (SPP) ubiquitin E3 ligase has also been shown to promote HDAC6 polyubiquitination and degradation (Tan et al. 2017). HDAC6 has been shown to modulate radiosensitivity in NSCLC by promoting CHK1 degradation (Moses et al. 2020). Ubiquitin ligase Parkin mutation, which causes early onset familial Parkinson's disease, catalyzes mitochondrial ubiquitination and promotes mitochondrial binding to HDAC6 and P62, leads to mitochondrial clearance (Lee et al. 2010). HDAC6 also regulates Hsp90 through the $\mathrm{ZnF}-\mathrm{UBP}$ domain. The main mechanism is the binding of ubiquitinated protein aggregates through the ubiquitin-binding functional domain of HDAC6. The binding of HDAC6 to ubiquitin leads to the dissociation of the HDAC6-P97/VCP complex, which further dissociates the Hsp90-HSF1 complex through the ATPase activity of HDAC6, and then HSF1 is activated (Boyault et al. 2007) (Fig. 3). HDAC6 protects cells from cytotoxic effects caused by abnormal protein folding and is crucial in biomedicine and in human disease, and in healthy development, it plays an irreplaceable role. It seems that the combined inhibition may lead to impressive therapeutic benefits based on the HDAC6-Hsp90 relationship.

\section{Development and application of HDAC6 and Hsp90 inhibitors based on their relationship}

HDAC6 and Hsp90 are widely investigated anticancer drug targets. Current data indicate that there has an extremely close interaction between HDAC6 and Hsp90 (Fig. 2). Their interdependence and participation in overlapping signaling networks make them ideal candidates for multitargeting approaches, particularly in cancer cells (Krämer et al. 2014). To date, there have been numerous reports to prove the effectiveness of the interaction between the two in clinical application. For example, Park, Younghee et al. demonstrated the prognostic value of the association between HDAC6 and Hsp90 in patients with early-stage breast cancer and proposed a new approach to target HDAC6 and Hsp90 in the clinical treatment of breast cancer (Park et al. 2015). In addition, several studies have reported the role of HDAC6 and Hsp90 in the treatment of prostate cancer. Basak, Shashwati et al. demonstrated a new mechanism by which genistein downregulates the 


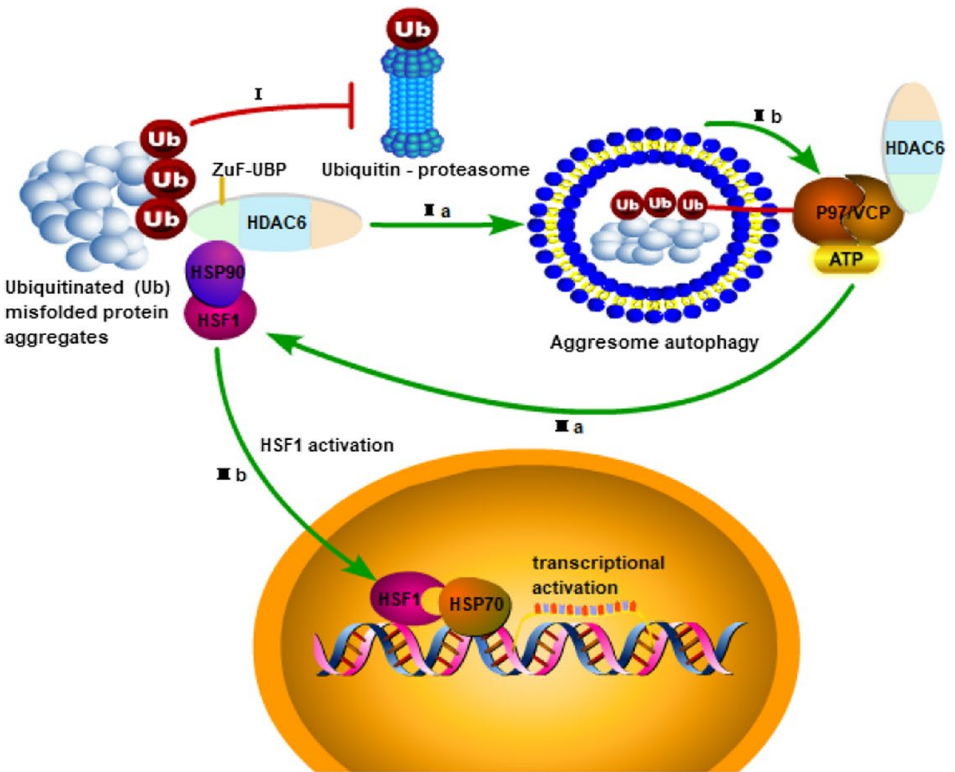

Fig. 3 Regulation Hsp90 by the ZnF-UBP of HDAC6

androgen receptor AR protein by inhibiting the chaperone function of HDAC6-Hsp90. This suggests that genistein may be used as a potential chemoprophylaxis for prostate cancer in combination with known inhibitors of HDAC6 and Hsp90 (Basak et al. 2008). HDAC6 regulates androgen receptor hypersensitivity and nuclear localization by regulating Hsp90 acetylation in castration-resistant prostate cancer, and targeting HDAC6 or in combination with other therapeutic approaches is a promising new strategy for the prevention and/or treatment of castration-resistant prostate cancer (Ai et al. 2009). It is also possible to optimize treatment by designing dual-acting compounds of AR and HDAC6 (Jadhavar et al. 2016). Inhibition of HDAC6 enhanced the binding of Hsp90 to 17-AAG in human leukemia cells (Rao et al. 2008). Therefore, combined with the information presented herein, exploring original and high-efficiency drugs or combined utilization targeting HDAC6 and/or Hsp90 is a hot research direction, especially for antitumor applications (Tao et al. 2018; Rosati et al. 2016; Seidel et al. 2016; Qu et al. 2012). Luca Pinzi developed and applied an integrated computational strategy to design dual inhibitors of HDAC6 and Hsp90, and some of them were able to selectively provide an increased level of acetylation of $\alpha$-tubulin; notably, two compounds also demonstrated a reduction in breast cancer cell proliferation (Pinzi et al. 2020).
Significant progress has also been made in the development of efficient and specific moderators of HDAC6, taking advantage of the latest and powerful protein degradation method PROTACs (An et al. 2019). In addition, the combination of inhibitors is also a noteworthy research direction (Yu et al. 2017). A phase I-II study of the histone deacetylase inhibitor vorinostat plus sequential weekly paclitaxel was undertaken for locally advanced breast cancer (Tu et al. 2014). In addition, excavating bispecific inhibitors is continuing. Ritu Ojha synthesized a series of 1-arylpyridine-hydroxamic acids, and their protein inhibitory and antitumor biological activities were evaluated. Compound 12(N1-(1-(2,4-dihydroxy-5-isopropylbenzoyl)indolin5-yl)-N8-hydroxyoctanediamide) showed significant inhibitory effects on both Hsp90 and HDAC6, as well as significant tumor cytotoxic effects (Ojha et al. 2018). Simultaneously, a series of 4,5,6,7-tetrahydro-isoxazolo-[4,5-c]-pyridines were synthesized, and most of them showed inhibitory activity against Hsp90. Moreover, a derivative bearing a hydroxamic acid residue bound to the $\mathrm{C}-3$ amide portion was found to inhibit both Hsp90 and HDAC6 (Baruchello et al. 2014). The aforementioned studies indicate that the search for dual inhibitors of Hsp90 and HDAC6 is a promising new direction for the development of novel antitumor drugs. 


\section{Conclusions}

In this paper, we mainly reviewed the structural characteristics and functions of HDAC6 and Hsp90, as well as the molecular mechanism of their interaction and the latest research progress, with the purpose of providing some insights into clinical drug development of HDAC6 and Hsp90. The interaction between HDAC6 and Hsp90 is closely related to the treatment of human malignant diseases, and the most common treatment is combined therapy with their specific inhibitors. Tremendous progress in basic research and clinical drug development and application has led to significant technological breakthroughs in major diseases such as tumors, viral infections, and imbalances in immune regulation.

As mentioned above, HDAC6-specific inhibitors are widely used in multiple myeloma, studies have shown that $\mathrm{As}_{2} \mathrm{O}_{3}$ plays an anti-multiple myeloma role by inhibiting the activity of HDAC6, promoting the acetylation of $\alpha$-tubulin, reducing the function of Hsp90, and leading to the inactivation of NF- $\kappa B$. This result provides an important insight into the molecular mechanism of $\mathrm{As}_{2} \mathrm{O}_{3}$ 's anti-myeloma activity, and provides theoretical support for HDAC6 to serve for clinical diseases. However, the study did not have clinical samples to support its conclusions ( $\mathrm{Qu}$ et al. 2012). In addition, HDAC6-specific inhibitors also used in neurodegenerative disease, the absence of a novel CHIP substrate, HDAC6, has been shown to mitigate the abnormal accumulation of Tau, while the absence of HDAC6 activity further enhances the efficacy of Hsp90 inhibitors, leading to tau degradation. The results suggest that HDAC6 is a key factor in regulating tau levels and suggest a multifaceted approach to treating neurodegenerative diseases caused by abnormal tau accumulation. However, the specific molecular mechanism of HDAC6-HSP90-Tau in this paper remains unknow. If clinical samples are available, the root will be convincing (Cook et al. 2012). Furthermore, HDAC6 inhibitors have potential application value in the treatment of glioblastoma, Zong-Yang Li et al. confirmed for the first time that ER stress-tolerance (ERST) occurred in Temozolomide (TMZ) resistant glioma cells. In addition, they found that the HDAC6 inhibitor Tubastatin A (TUB) may overcome ERST through two cellular signaling pathways: enhancing the P97/VCP-mediated ubiquitin-proteasome degradation system, and inhibiting the HDAC6-mediated autophagy pathway. The study demonstrates that HDAC6 inhibitors are a potential target for the treatment of gliomas, and demonstrates that the combination of TMZ and TUB may be an effective strategy to facilitate the development of new clinical therapies. However, the authors should assist in verifying the results by directly knocking out the ubiquitin-binding domain of HDAC6 (Zheng and Wang 2010). Last but not least, HDAC6 has been widely reported in cancer therapy (Li et al. 2018). Hsp90 has become a potential target for the treatment of a variety of diseases, including Alzheimer's disease and Parkinson's disease. Although a large number of studies have been carried out in this field, the specific molecular mechanism of their action is still unclear. Therefore, it is very important to develop innovative, specific and efficient Hsp90 inhibitor molecules for clinical application (Alam et al. 2017). In conclusion, various studies using HDAC6 inhibitors alone or in combination with other drugs provide a strong scientific basis for the clinical development of these new drugs in human diseases.

To date, limited by low efficacy, water solubility, and lipid solubility, toxicity, or acquired drug resistance, none of the Hsp90 inhibitors have received clinical approval (Oh et al. 2021). The ever-growing studies reporting the interaction between HDAC6 and Hsp90 and overlapping networks are helpful to further understand the molecular regulation mechanism. Since HDAC6 and Hsp90 have vital functions in the regulation of multifarious signaling pathway, gene expression, protein stability and other cellular functions, the development and research of HDAC6-Hsp90 inhibitors have gradually increased, and some inhibitors have entered the clinical research stage, although the two targets share rare homology. However, this is not enough, and we must seek solutions and develop strategies from the commanding height of innovation, such as the use of nanomaterial delivery inhibitors to solve the problems of water solubility and cytotoxicity to achieve the purpose of safety and efficiency. On the basis of a full understanding of the specific mechanism and regulatory relationship between their interaction, suitably matched and effective dual inhibitors will be designed and synthesized to create clinical treatments.

\section{Acknowledgements \\ Not applicable.}

\section{Authors' contributions}

JX and PL outlined and edited the manuscript; YW and KK designed the study and revised the manuscript; YW, XS and LH participated in data analysis and drafting. All authors declare no conflicts of interest associated with this manuscript. All authors read and approved the final manuscript.

\section{Funding}

The research described herein was supported by grants from the National Natural Science Foundation of China [Grant No. 82072274] and Guangdong Natural Science Foundation [Grant No. 2019A1515010046].

\section{Declarations}

Ethical approval and consent to participate Not applicable.

Consent for publication

All authors agreed to this publication. 


\section{Competing interests}

The authors declare that they have no competing interests.

\section{Author details}

${ }^{1}$ College of Life Science and Technology, Guangzhou Jinan Biomedicine Research and Development Center, Jinan University, Guangzhou, China. ${ }^{2} \mathrm{Col}-$ lege of Pharmacy, Jinan University, Guangzhou, China. ${ }^{3}$ Department of Clinical Research Pharmacy, Graduate School of Biomedical Sciences, Nagasaki University, Nagasaki, Japan.

Received: 28 June 2021 Accepted: 7 September 2021

\section{Published online: 16 September 2021}

\section{References}

Ai J, Wang Y, Dar JA, Liu J, Liu L, Nelson JB, et al. HDAC6 regulates androgen receptor hypersensitivity and nuclear localization via modulating Hsp90 acetylation in castration-resistant prostate cancer. Mol Endocrinol (baltimore, Md). 2009:23(12):1963-72

Alam Q, Alam MZ, Sait KHW, Anfinan N, Noorwali AW, Kamal MA, et al. Translational shift of HSP90 as a novel therapeutic target from cancer to neurodegenerative disorders: an emerging trend in the cure of Alzheimer's and Parkinson's diseases. Curr Drug Metab. 2017;18(9):868-76.

An Z, Lv W, Su S, Wu W, Rao Y. Developing potent PROTACs tools for selective degradation of HDAC6 protein. Protein Cell. 2019;10(8):606-9.

Bagchi R, Weeks K. Histone deacetylases in cardiovascular and metabolic diseases. J Mol Cell Cardiol. 2019;130:151-9.

Bali P, Pranpat M, Bradner J, Balasis M, Fiskus W, Guo F, et al. Inhibition of histone deacetylase 6 acetylates and disrupts the chaperone function of heat shock protein 90: a novel basis for antileukemia activity of histone deacetylase inhibitors. J Biol Chem. 2005;280(29):26729-34.

Banerjee M, Hatial I, Keegan BM, Blagg BSJ. Assay design and development strategies for finding Hsp90 inhibitors and their role in human diseases. Pharmacol Ther. 2020:221:107747.

Baruchello R, Simoni D, Marchetti P, Rondanin R, Mangiola S, Costantini C, et al. 4,5,6,7-Tetrahydro-isoxazolo-[4,5-c]-pyridines as a new class of cytotoxic Hsp90 inhibitors. Eur J Med Chem. 2014;76:53-60.

Basak S, Pookot D, Noonan EJ, Dahiya R. Genistein down-regulates androgen receptor by modulating HDAC6-Hsp90 chaperone function. Mol Cancer Ther. 2008;7(10):3195-202.

Benoy V, Vanden Berghe P, Jarpe M, Van Damme P, Robberecht W, Van Den Bosch L. Development of improved HDAC6 inhibitors as pharmacological therapy for axonal Charcot-Marie-Tooth disease. Neurotherapeutics. 2017;14(2):417-28.

Boyault C, Zhang Y, Fritah S, Caron C, Gilquin B, Kwon SH, et al. HDAC6 controls major cell response pathways to cytotoxic accumulation of protein aggregates. Genes Dev. 2007;21(17):2172-81.

Calderwood S, Mambula S, Gray P, Theriault J. Extracellular heat shock proteins in cell signaling. FEBS Lett. 2007;581(19):3689-94.

Chan KC, Ting CM, Chan PS, Lo MC, Lo KW, Curry JE, et al. A novel Hsp90 inhibitor AT13387 induces senescence in EBV-positive nasopharyngeal carcinoma cells and suppresses tumor formation. Mol Cancer. 2013;12(1):128.

Chaudhury S, Keegan BM, Blagg BSJ. The role and therapeutic potential of Hsp90, Hsp70, and smaller heat shock proteins in peripheral and central neuropathies. Med Res Rev. 2021;41(1):202-22.

Chen B, Piel WH, Gui L, Bruford E, Monteiro A. The HSP90 family of genes in the human genome: insights into their divergence and evolution. Genomics. 2005;86(6):627-37.

Chen B, Zhong D, Monteiro A. Comparative genomics and evolution of the HSP90 family of genes across all kingdoms of organisms. BMC Genomics. 2006:7:156.

Choudhary C, Kumar C, Gnad F, Nielsen M, Rehman M, Walther T, et al. Lysine acetylation targets protein complexes and co-regulates major cellular functions. Science (new York, NY). 2009;325(5942):834-40.

Cook C, Gendron TF, Scheffel K, Carlomagno Y, Dunmore J, DeTure M, et al. Loss of HDAC6, a novel CHIP substrate, alleviates abnormal tau accumulation. Hum Mol Genet. 2012;21(13):2936-45.

Cosenza M, Pozzi S. The therapeutic strategy of HDAC6 inhibitors in lymphoproliferative disease. Int J Mol Sci. 2018;19(8):2337. de Ruijter AJ, van Gennip AH, Caron HN, Kemp S, van Kuilenburg AB. Histone deacetylases (HDACs): characterization of the classical HDAC family. Biochem J. 2003:370(Pt 3):737-49.

Espallergues J, Teegarden SL, Veerakumar A, Boulden J, Challis C, Jochems $J$, et al. HDAC6 regulates glucocorticoid receptor signaling in serotonin pathways with critical impact on stress resilience. J Neurosci. 2012;32(13):4400-16.

Fiskus W, Ren Y, Mohapatra A, Bali P, Mandawat A, Rao R, et al. Hydroxamic acid analogue histone deacetylase inhibitors attenuate estrogen receptoralpha levels and transcriptional activity: a result of hyperacetylation and inhibition of chaperone function of heat shock protein 90. Clin Cancer Res. 2007:13(16):4882-90.

Fusco C, Micale L, Egorov M, Monti M, D'Addetta EV, Augello B, et al. The E3-ubiquitin ligase TRIM50 interacts with HDAC6 and p62, and promotes the sequestration and clearance of ubiquitinated proteins into the aggresome. PLoS One. 2012;7(7):e40440.

Gao YS, Hubbert CC, Lu J, Lee YS, Lee JY, Yao TP. Histone deacetylase 6 regulates growth factor-induced actin remodeling and endocytosis. Mol Cell Biol. 2007;27(24):8637-47

Gibbs A, Schwartzman J, Deng V, Alumkal J. Sulforaphane destabilizes the androgen receptor in prostate cancer cells by inactivating histone deacetylase 6. Proc Natl Acad Sci U S A. 2009;106(39):16663-8.

Glozak MA, Sengupta N, Zhang X, Seto E. Acetylation and deacetylation of non-histone proteins. Gene. 2005:363:15-23.

Gravina GL, Marampon F, Muzi P, Mancini A, Piccolella M, Negri-Cesi P, et al. PXD101 potentiates hormonal therapy and prevents the onset of castration-resistant phenotype modulating androgen receptor, HSP90, and CRM1 in preclinical models of prostate cancer. Endocr Relat Cancer. 2013:20(3):321-37

Hsieh YL, Tu HJ, Pan SL, Liou JP, Yang CR. Anti-metastatic activity of MPTOG211, a novel HDAC6 inhibitor, in human breast cancer cells in vitro and in vivo. Biochim Biophys Acta Mol Cell Res. 2019;1866(6):992-1003.

Hu WY, Xu L, Chen B, Ou S, Muzzarelli KM, Hu DP, et al. Targeting prostate cancer cells with enzalutamide-HDAC inhibitor hybrid drug 2-75. Prostate. 2019;79(10):1166-79

Hubbert C, Guardiola A, Shao R, Kawaguchi Y, Ito A, Nixon A, et al. HDAC6 is a microtubule-associated deacetylase. Nature. 2002;417(6887):455-8.

Iwata A, Riley BE, Johnston JA, Kopito RR. HDAC6 and microtubules are required for autophagic degradation of aggregated huntingtin. J Biol Chem. 2005;280(48):40282-92.

Jadhavar PS, Ramachandran SA, Riquelme E, Gupta A, Quinn KP, Shivakumar D, et al. Targeting prostate cancer with compounds possessing dual activity as androgen receptor antagonists and HDAC6 inhibitors. Bioorg Med Chem Lett. 2016;26(21):5222-8.

Jochems J, Teegarden SL, Chen Y, Boulden J, Challis C, Ben-Dor GA, et al. Enhancement of stress resilience through histone deacetylase 6-mediated regulation of glucocorticoid receptor chaperone dynamics. Biol Psychiatry. 2015;77(4):345-55

Kalveram B, Schmidtke G, Groettrup M. The ubiquitin-like modifier FAT10 interacts with HDAC6 and localizes to aggresomes under proteasome inhibition. J Cell Sci. 2008:121(Pt 24):4079-88.

Kamemura K, Ogawa M, Ohkubo S, Ohtsuka Y, Shitara Y, Komiya T, et al. Depression of mitochondrial metabolism by downregulation of cytoplasmic deacetylase, HDAC6. FEBS Lett. 2012;586(9):1379-83.

Kekatpure VD, Dannenberg AJ, Subbaramaiah K. HDAC6 modulates Hsp90 chaperone activity and regulates activation of aryl hydrocarbon receptor signaling. J Biol Chem. 2009;284(12):7436-45.

Kim IA, No M, Lee JM, Shin JH, Oh JS, Choi EJ, et al. Epigenetic modulation of radiation response in human cancer cells with activated EGFR or HER-2 signaling: potential role of histone deacetylase 6. Radiother Oncol. 2009;92(1):125-32.

Kovacs JJ, Murphy PJ, Gaillard S, Zhao X, Wu JT, Nicchitta CV, et al. HDAC6 regulates $\mathrm{Hsp} 90$ acetylation and chaperone-dependent activation of glucocorticoid receptor. Mol Cell. 2005;18(5):601-7.

Kovacs JJ, Cohen TJ, Yao TP. Chaperoning steroid hormone signaling via reversible acetylation. Nucl Recept Signal. 2005;3:e004.

Krämer OH, Mahboobi S, Sellmer A. Drugging the HDAC6-HSP90 interplay in malignant cells. Trends Pharmacol Sci. 2014;35(10):501-9.

Lebret T, Watson R, Molinié V, O'Neill A, Gabriel C, Fitzpatrick J, et al. Heat shock proteins HSP27, HSP60, HSP70, and HSP90: expression in bladder carcinoma. Cancer. 2003:98(5):970-7. 
Lee SM, Bae JH, Kim MJ, Lee HS, Lee MK, Chung BS, et al. Bcr-Abl-independent imatinib-resistant K562 cells show aberrant protein acetylation and increased sensitivity to histone deacetylase inhibitors. J Pharmacol Exp Ther. 2007:322(3):1084-92.

Lee JY, Nagano Y, Taylor JP, Lim KL, Yao TP. Disease-causing mutations in parkin impair mitochondrial ubiquitination, aggregation, and HDAC6-dependent mitophagy. J Cell Biol. 2010;189(4):671-9.

Lee JB, Wei J, Liu W, Cheng J, Feng J, Yan Z. Histone deacetylase 6 gates the synaptic action of acute stress in prefrontal cortex. J Physiol. 2012;590(7):1535-46.

Lee JY, Kuo CW, Tsai SL, Cheng SM, Chen SH, Chan HH, et al. Inhibition of HDAC3- and HDAC6-promoted survivin expression plays an important role in SAHA-induced autophagy and viability reduction in breast cancer cells. Front Pharmacol. 2016;7:81.

Li D, Marchenko ND, Moll UM. SAHA shows preferential cytotoxicity in mutant p53 cancer cells by destabilizing mutant p53 through inhibition of the HDAC6-Hsp90 chaperone axis. Cell Death Differ. 2011;18(12):1904-13.

Li Y, Shin D, Kwon SH. Histone deacetylase 6 plays a role as a distinct regulator of diverse cellular processes. FEBS J. 2013;280(3):775-93.

Li ZY, Zhang C, Zhang Y, Chen L, Chen BD, Li QZ, et al. A novel HDAC6 inhibitor Tubastatin A: controls HDAC6-p97/NCP-mediated ubiquitinationautophagy turnover and reverses Temozolomide-induced ER stresstolerance in GBM cells. Cancer Lett. 2017;391:89-99.

Li T, Zhang C, Hassan S, Liu X, Song F, Chen K, et al. Histone deacetylase 6 in cancer. J Hematol Oncol. 2018;11(1):111.

Lin TY, Fenger J, Murahari S, Bear MD, Kulp SK, Wang D, et al. AR-42, a novel HDAC inhibitor, exhibits biologic activity against malignant mast cell lines via down-regulation of constitutively activated Kit. Blood. 2010;115(21):4217-25

Liu F, Wang L, Yi S, Liu Q, Xu X, Su M. Clinical and biological significances of heat shock protein 90 (Hsp90) in human nasopharyngeal carcinoma cells and anti-cancer effects of Hsp90 inhibitor. Biomed Pharmacother. 2019;120:109533.

Lu CY, Chang YC, Hua CH, Chuang C, Huang SH, Kung SH, et al. Tubacin, an HDAC6 selective inhibitor, reduces the replication of the japanese encephalitis virus via the decrease of viral RNA synthesis. Int J Mol Sci. 2017;18(5):954.

Luengo TM, Mayer MP, Rüdiger SGD. The Hsp70-Hsp90 chaperone cascade in protein folding. Trends Cell Biol. 2019;29(2):164-77.

Matsuyama A, Shimazu T, Sumida Y, Saito A, Yoshimatsu Y, Seigneurin-Berny D, et al. In vivo destabilization of dynamic microtubules by HDAC6-mediated deacetylation. Embo J. 2002;21 (24):6820-31.

Matthias P, Yoshida M, Khochbin S. HDAC6 a new cellular stress surveillance factor. Cell Cycle. 2008;7(1):7-10.

Mellatyar H, Talaei S, Pilehvar-Soltanahmadi Y, Barzegar A, Akbarzadeh A, Shahabi A, et al. Targeted cancer therapy through 17-DMAG as an Hsp90 inhibitor: overview and current state of the art. Biomed Pharmacother. 2018;102:608-17.

Meng Q, Chen X, Sun L, Zhao C, Sui G, Cai L. Carbamazepine promotes Her-2 protein degradation in breast cancer cells by modulating HDAC6 activity and acetylation of Hsp90. Mol Cell Biochem. 2011;348(1-2):165-71.

Morgan M, Shilatifard A. Reevaluating the roles of histone-modifying enzymes and their associated chromatin modifications in transcriptional regulation. Nat Genet. 2020;52(12):1271-81.

Moses N, Zhang M, Wu JY, Hu C, Xiang S, Geng X, et al. HDAC6 regulates radiosensitivity of non-small cell lung cancer by promoting degradation of Chk1. Cells. 2020;9(10):2237.

Murphy PJ, Morishima Y, Kovacs JJ, Yao TP, Pratt WB. Regulation of the dynamics of hsp90 action on the glucocorticoid receptor by acetylation/deacetylation of the chaperone. J Biol Chem. 2005:280(40):33792-9.

Narita T, Weinert BT, Choudhary C. Functions and mechanisms of non-histone protein acetylation. Nat Rev Mol Cell Biol. 2019;20(3):156-74.

New M, Olzscha H, Liu G, Khan O, Stimson L, McGouran J, et al. A regulatory circuit that involves HR23B and HDAC6 governs the biological response to HDAC inhibitors. Cell Death Differ. 2013;20(10):1306-16.

Newbold A, Matthews GM, Bots M, Cluse LA, Clarke CJ, Banks KM, et al. Molecular and biologic analysis of histone deacetylase inhibitors with diverse specificities. Mol Cancer Ther. 2013;12(12):2709-21.

Oh YJ, Park SY, Seo YH. Selective targeting of cancer cells using a hydrogen peroxide-activated Hsp90 inhibitor. Bioorg Chem. 2021;115:105195.
Ojha R, Huang HL, HuangFu WC, Wu YW, Nepali K, Lai MJ, et al. 1-Aroylindolinehydroxamic acids as anticancer agents, inhibitors of HSP90 and HDAC. Eur J Med Chem. 2018:150:667-77.

Pai JT, Hsu CY, Hua KT, Yu SY, Huang CY, Chen CN, et al. NBM-T-BBX-OSO1, semisynthesized from osthole, induced G1 growth arrest through HDAC6 inhibition in lung cancer cells. Molecules. 2015;20(5):8000-19.

Pai JT, Hsu CY, Hsieh YS, Tsai TY, Hua KT, Weng MS. Suppressing migration and invasion of $\mathrm{H} 1299$ lung cancer cells by honokiol through disrupting expression of an HDAC6-mediated matrix metalloproteinase 9. Food Sci Nutr. 2020;8(3):1534-45.

Pandey UB, Batlevi Y, Baehrecke EH, Taylor JP. HDAC6 at the intersection of autophagy, the ubiquitin-proteasome system and neurodegeneration. Autophagy. 2007;3(6):643-5.

Park JH, Kim SH, Choi MC, Lee J, Oh DY, Im SA, et al. Class II histone deacetylases play pivotal roles in heat shock protein 90 -mediated proteasomal degradation of vascular endothelial growth factor receptors. Biochem Biophys Res Commun. 2008;368(2):318-22.

Park Y, Lee KS, Park SY, Kim JH, Kang EY, Kim SW, et al. Potential prognostic value of histone deacetylase 6 and acetylated heat-shock protein 90 in early-stage breast cancer. J Breast Cancer. 2015;18(3):249-55.

Pinzi L, Benedetti R, Altucci L, Rastelli G. Design of dual inhibitors of histone deacetylase 6 and heat shock protein 90. ACS Omega. 2020;5(20):11473-80.

Qu X, Du J, Zhang C, Fu W, Xi H, Zou J, et al. Arsenic trioxide exerts antimyeloma effects by inhibiting activity in the cytoplasmic substrates of histone deacetylase 6. PLoS One. 2012;7(2):e32215.

Rao R, Fiskus W, Yang Y, Lee P, Joshi R, Fernandez P, et al. HDAC6 inhibition enhances 17-AAG-mediated abrogation of hsp90 chaperone function in human leukemia cells. Blood. 2008:112(5):1886-93.

Rosati R, Chen B, Patki M, McFall T, Ou S, Heath E, et al. Hybrid enzalutamide derivatives with histone deacetylase inhibitor activity decrease heat shock protein 90 and androgen receptor levels and inhibit viability in enzalutamide-resistant C4-2 prostate cancer cells. Mol Pharmacol. 2016;90(3):225-37.

Ryu HW, Won HR, Lee DH, Kwon SH. HDAC6 regulates sensitivity to cell death in response to stress and post-stress recovery. Cell Stress Chaperones. 2017a;22(2):253-61.

Ryu HW, Shin DH, Lee DH, Choi J, Han G, Lee KY, et al. HDAC6 deacetylates p53 at lysines 381/382 and differentially coordinates p53-induced apoptosis. Cancer Lett. 2017b;391:162-71.

Schoepflin ZR, Shapiro IM, Risbud MV. Class I and Ila HDACs mediate HIF-1a stability through PHD2-dependent mechanism, while HDAC6, a Class IIb Member, promotes HIF-1a transcriptional activity in nucleus pulposus cells of the intervertebral disc. J Bone Miner Res. 2016;31 (6):1287-99.

Schulz R, Marchenko ND, Holembowski L, Fingerle-Rowson G, Pesic M, Zender $L$, et al. Inhibiting the HSP9O chaperone destabilizes macrophage migration inhibitory factor and thereby inhibits breast tumor progression. J Exp Med. 2012;209(2):275-89.

Scroggins BT, Robzyk K, Wang D, Marcu MG, Tsutsumi S, Beebe K, et al. An acetylation site in the middle domain of $\mathrm{Hsp} 90$ regulates chaperone function. Mol Cell. 2007;25(1):151-9.

Seidel C, Schnekenburger M, Dicato M, Diederich M. Histone deacetylase 6 in health and disease. Epigenomics. 2015:7(1):103-18.

Seidel C, Schnekenburger M, Mazumder A, Teiten MH, Kirsch G, Dicato M, et al. 4-Hydroxybenzoic acid derivatives as HDAC6-specific inhibitors modulating microtubular structure and HSP90a chaperone activity against prostate cancer. Biochem Pharmacol. 2016:99:31-52.

Selenica ML, Benner L, Housley SB, Manchec B, Lee DC, Nash KR, et al. Histone deacetylase 6 inhibition improves memory and reduces total tau levels in a mouse model of tau deposition. Alzheimers Res Ther. 2014;6(1):12.

Simões-Pires C, Zwick V, Nurisso A, Schenker E, Carrupt P, Cuendet M. HDAC6 as a target for neurodegenerative diseases: what makes it different from the other HDACs? Mol Neurodegener. 2013;8:7.

Sreedharan R, Van Why S. Heat shock proteins in the kidney. Pediatric Nephrology (berlin, Germany). 2016;31(10):1561-70.

Tan Y, Ci Y, Dai X, Wu F, Guo J, Liu D, et al. Cullin 3SPOP ubiquitin E3 ligase promotes the poly-ubiquitination and degradation of HDAC6. Oncotarget. 2017:8(29):47890-901.

Tao H, Chen YY, Sun ZW, Chen HL, Chen M. Silence of HDAC6 suppressed esophageal squamous cell carcinoma proliferation and 
migration by disrupting chaperone function of HSP90. J Cell Biochem. 2018;119(8):6623-32.

Tsai RY, Cheng YC, Wong CS. (+)-Naloxone inhibits morphine-induced chemotaxis via prevention of heat shock protein 90 cleavage in microglia. J Formos Med Assoc. 2015;114(5):446-55.

Tu Y, Hershman D, Bhalla K, Fiskus W, Pellegrino C, Andreopoulou E, et al. A phase I-II study of the histone deacetylase inhibitor vorinostat plus sequential weekly paclitaxel and doxorubicin-cyclophosphamide in locally advanced breast cancer. Breast Cancer Res Treat. 2014;146(1):145-52.

Verdel A, Khochbin S. Identification of a new family of higher eukaryotic histone deacetylases. Coordinate expression of differentiation-dependent chromatin modifiers. J Biol Chem. 1999;274(4):2440-5.

Verheyen A, Peeraer E, Nuydens R, Dhondt J, Poesen K, Pintelon I, et al. Systemic anti-vascular endothelial growth factor therapies induce a painful sensory neuropathy. Brain. 2012;135(Pt 9):2629-41.

Yan J. Interplay between HDAC6 and its interacting partners: essential roles in the aggresome-autophagy pathway and neurodegenerative diseases DNA Cell Biol. 2014;33(9):567-80.

Yan J, Seibenhener ML, Calderilla-Barbosa L, Diaz-Meco MT, Moscat J, Jiang J, et al. SQSTM1/p62 interacts with HDAC6 and regulates deacetylase activity. PLoS One. 2013:8(9):e76016.

Yao ZG, Li WH, Hua F, Cheng HX, Zhao MQ, Sun XC, et al. LBH589 inhibits glioblastoma growth and angiogenesis through suppression of HIF-1a expression. J Neuropathol Exp Neurol. 2017;76(12):1000-7.
Yu S, Cai X, Wu C, Liu Y, Zhang J, Gong X, et al. Targeting HSP90-HDAC6 regulating network implicates precision treatment of breast cancer. Int J Biol Sci. 2017:13(4):505-17.

Zhang D, Li J, Costa M, Gao J, Huang C. JNK1 mediates degradation HIF-1alpha by a VHL-independent mechanism that involves the chaperones Hsp90/ Hsp70. Cancer Res. 2010;70(2):813-23.

Zhang L, Ogden A, Aneja R, Zhou J. Diverse roles of HDAC6 in viral infection: implications for antiviral therapy. Pharmacol Ther. 2016;164:120-5.

Zheng Q, Wang X. Autophagy and the ubiquitin-proteasome system in cardiac dysfunction. Panminerva Med. 2010;52(1):9-25.

Zheng K, Jiang Y, He Z, Kitazato K, Wang Y. Cellular defence or viral assist: the dilemma of HDAC6. J Gen Virol. 2017;98(3):322-37.

Zheng Y, Zhu G, Tang Y, Yan J, Han S, Yin J, et al. HDAC6, a novel cargo for autophagic clearance of stress granules, mediates the repression of the Type I interferon response during coxsackievirus A16 infection. Front Microbiol. 2020;11:78.

Zhu K, Zhang Y, Zhang J, Zhou F, Zhang L, Wang S, et al. Acetylation of Hsp90 reverses dexamethasone-mediated inhibition of insulin secretion. Toxicol Lett. 2020;320:19-27.

\section{Publisher's Note}

Springer Nature remains neutral with regard to jurisdictional claims in published maps and institutional affiliations.
Ready to submit your research? Choose BMC and benefit from:

- fast, convenient online submission

- thorough peer review by experienced researchers in your field

- rapid publication on acceptance

- support for research data, including large and complex data types

- gold Open Access which fosters wider collaboration and increased citations

- maximum visibility for your research: over 100M website views per year

At BMC, research is always in progress.

Learn more biomedcentral.com/submissions 